\title{
PM2.5 promotes apoptosis of human epidermal melanocytes through promoting oxidative damage and autophagy
}

\author{
Pin Liang ${ }^{1}$, Xinli Xing ${ }^{2}$, Jianbo Wu ${ }^{1}$, Jiquan Song ${ }^{1}$ and Qin Liu ${ }^{1}$ \\ ${ }^{1}$ Department of Dermatology, Zhongnan Hospital of Wuhan University, Wuchang District, Wuhan, Hubei, P.R. China \\ ${ }^{2}$ School of Environmental Studies, China University of Geosciences, Wuhan, Hubei, P.R. China
}

\begin{abstract}
Pollutants such as PM2.5 are polluting the environment seriously, causing numerous health problems. However, the skin toxicity caused by PM2.5 has been little reported so far. CCK- 8 was used to test the effects of PM2.5 on melanin cell proliferation. The effect of PM2.5 on melanocyte apoptosis was detected by flow cytometry. ELISA was used to detect the expression of oxidative stress-related factors, including reactive oxygen species (ROS). The expression of autophagosomes was detected by MDC immunohistochemical staining, and Western blot was used to detect the expression of autophagy marker LC3II/I. With the increasing concentrations of PM2.5, the proliferation rate and apoptosis rate of melanocytes decreased significantly, meanwhile the expression of oxidative stressrelated factors ROS, was obviously increased. The expression of LC3II/I induced by PM2.5 venom was higher than that of the control group in a concentration-dependent manner. However, there was no statistically significant difference between the water-soluble components of PM2.5 and the water-insoluble ones. PM2.5 can inhibit the proliferation of melanocytes and induce their apoptosis, which may be related to the oxidative damage of PM2.5. PM2.5 also induced autophagy in melanocytes, which is obviously correlated with its concentration. The mechanism may be a self-protective response of cells to oxidative stress injury and apoptosis.
\end{abstract}

Key words: PM2.5 - Apoptosis — Pigment diseases - Oxidative damage — Autophagy

\section{Introduction}

The change of skin color caused by the decrease or increase of pigments is called pigmented dermatosis. Lack of melanin will contribute to the reduction of pigment, and the general or localized whitening of the skin has become a major characteristic of this disease (Glassman 2011). The diseases caused by hyperpigmentation or an increase in nevus cells, such as freckles or pigment, are called melanin diseases (Kupsa et al. 2019). Nowadays, the incidence of pigment skin diseases is increasing, and people who are suffering from these diseases are at younger ages. What's more, environmental factors also participate in the pathogenesis of melanin diseases.

PM2.5 refers to particles in the air whose dynamic diameter is less than $2.5 \mu \mathrm{m}$, which can go into the alveoli of the

Correspondence to: Qin Liu, Department of Dermatology, Zhongnan Hospital of Wuhan University, No. 169 DongHu Road, Wuchang District,Wuhan, Hubei 430071, P.R. China

E-mail: liuqin0206@yeah.net human body; such particles are also called inhalable lung particles (Liu et al. 2020). Despite PM2.5 is smaller than PM10 in particle size, it possesses a larger relative surface area, making it easier to absorb and bind to toxically heavy metal elements, and acidic oxides such as viruses and bacteria in the air will seriously affect the health of human and other organisms (Galvao et al. 2020).

In recent years, more and more epidemiological and toxicological studies have shown that the exposure of PM2.5 in the air is related to the incidence of a variety of diseases of residents, such as cardiovascular system (Mannucci et al. 2019), reproductive system (Yang et al. 2019), blood system (Zhang et al. 2019), immune system (Wan et al. 2019), respiratory system (Yang et al. 2020), etc. Some studies have also shown that it can sometimes induce cancer in severe cases (Ning et al. 2019; Su et al. 2019; Wang et al. 2019b). Existing studies about PM2.5 mainly focus on the damage of PM2.5 to respiratory system and cardiovascular system, while there are few studies on its toxic effect on skin. 
In this paper, we treated and cultured human epidermal melanocytes in vitro with different concentrations and components of PM2.5, and studied the possible mechanism of PM2.5's toxic effect on human skin by detecting oxidative damage and autophagy levels of cells.

\section{Materials and Methods}

\section{Cell culture}

Human epidermal melanocytes were obtained from the American Type Culture Collection (ATCC, Manassas, VA, USA). They were routinely cultured in DMEM (Gibco; Thermo Fisher Scientific, Inc.) high-sugar medium containing $10 \%$ FBS (BI), and placed in a cell incubator at $37^{\circ} \mathrm{C}$ relatively saturated with $5 \% \mathrm{CO}_{2}$ humidity. Then, cells in logarithmic stage with good growth state were selected for the experiment.

\section{Preparation of PM2.5 toxic solution}

PM2.5 was collected from the air in Huanggang city, Hubei province. Under aseptic conditions, the filter membrane containing PM2.5 was immersed in deionized water and subjected to ultrasonic concussion for 3 times, each time for $30 \mathrm{~min}$, and PM2.5 was removed. Then PM2.5 was freeze-dried into $\mathrm{PM} 2.5$ powder and stored in the $-80^{\circ} \mathrm{C}$ refrigerator for later use. PM2.5 powder was added into serum-free medium to form mother liquor of $1000 \mu \mathrm{g} / \mathrm{ml}$, and the mixture was thoroughly mixed and centrifuged at $13000 \times g$ for $30 \mathrm{~min}$, then the supernatant with the concentration of $1000 \mu \mathrm{g} / \mathrm{ml}$ was taken as PM2.5 water-soluble substances stock solution (water-soluble extracts, hereinafter referred to as W-PM2.5). Sediment concentration of dissolved isometric DMSO with the concentration of $1000 \mu \mathrm{g} / \mathrm{ml}$ was taken as PM2.5 water-insoluble substances stock solution (non-water-soluble extracts, hereinafter referred to as the NW-PM2.5). Before the treatment, the cells were divided into the Control group, W-PM2.5 group (1, 10, 100 and $250 \mu \mathrm{g} / \mathrm{ml}$ of W-PM2.5), and NW-PM2.5 poisoning venom intervention group $(1,10,100$ and $250 \mu \mathrm{g} / \mathrm{ml}$ of NW-PM2.5). For routine culture of melanocytes, $2 \mathrm{ml}$ complete medium was added to the control group, and $2 \mathrm{ml} \mathrm{W-PM2.5}$ and NW-PM2.5 toxic solution of preset concentration was added to the experimental group, respectively, and incubated in the cell incubator for $48 \mathrm{~h}$ for the following experiments.

\section{Cell counting Kit-8 (CCK-8) assay}

We divided the cells into Control group, DMSO group (DMEM+DMSO of the same amount as $250 \mu \mathrm{g} / \mathrm{ml}$ or $100 \mu \mathrm{g} / \mathrm{ml}$ of $\mathrm{NW}-\mathrm{PM} 2.5), 250 \mu \mathrm{g} / \mathrm{ml}$ and $100 \mu \mathrm{g} / \mathrm{ml}$ groups $(250 \mu \mathrm{g} / \mathrm{ml}$ and $100 \mu \mathrm{g} / \mathrm{ml}$ of NW-PM2.5, respectively); then CCK-8 technology detected the cell survival rate. Cells in logarithmic growth phase were placed in a 96-well plate $\left(10^{3}\right.$ cells/well), then the cells are treated respectively. After $0,24,48,72$ h of continuous culture, 101 CCK-8 solution was added into all wells and incubated in the incubator for 4 hours. The absorption value of $450 \mathrm{~nm}$ was determined by microplate (Bio-Rad, CA, USA) and the inhibition rate was calculated: Inhibition rate $\%=$ (control group - experimental group $) /($ control group - blank group) $\times 100 \%$.

\section{Flow cytometry}

In order to prepare cell suspension, cells in the six-well plate were collected. They were washed and diluted in a binding buffer with a $300 \mu$ pre-cooled PBS once. $5 \mu$ Annexin V-FITC (fluorescein-5-isothiocyanate) was added into the binding buffer, and the mixture was then incubated in darkness for $10 \mathrm{~min}$. Then $5 \mu \mathrm{l}$ propidium iodide (PI) was added, and the mixture was then incubated in darkness for $5 \mathrm{~min}$. Flow cytometer (FACSCalibur; Becton-Dickinson, Franklin Lakes, NJ, USA) was used to detect the activity of Annexin $\mathrm{V} / \mathrm{PI}$ in $1 \mathrm{~h}$, and corresponding channels were selected for observation and quantified by Flow Jo software (version 7.6.1; Flow Jo LLC, Ashland, OR, USA).

\section{Reactive oxygen species (ROS) assay}

ROS levels of cells were detected using a fluorescent probe, 2',7'-dichlorodihydrofluorescein (DCHF) (Sigma), which could be rapidly oxidized into the highly fluorescent 2',7'-dichlorofluorescein (DCF) in the presence of intracellular reactive oxygen species (ROS). ROS expression at $488 \mathrm{~nm}$ was analyzed by flow cytometry (FACSC alibur; BectonDickinson, Franklin Lakes, NJ, USA). The amount of ROS was quantified as the relative fluorescence intensity of DCF per cell in the scan area.

\section{Monodansylcadaverine (MDC) staining}

Cell resuspension solution was collected and centrifuged at a condition of $1500 \mathrm{rpm}$ for $5 \mathrm{~min}$. After washing in the wash buffer, the cells were resuspended and the cell concentration was adjusted to $10^{6} / \mathrm{ml}$. The cell suspension of $90 \mu \mathrm{l}$ was added into the new EP tube, and $10 \mu \mathrm{l}$ MDC dye liquor was added and gently mixed. After staining at room temperature and out of light for $30 \mathrm{~min}$, cells were collected and washed by Wash Buffer for 3 times, and then resuspended by $100 \mu \mathrm{l}$ collection Buffer. The suspended droplets were added to the glass slide, covered with glass slide, and placed under a fluorescence microscope (Leica, Germany) for observation and photographing. The fluo- 
rescence intensity of each marker was quantified using the Image J (1.48. version) software (National Institutes of Health, Bethesda).

\section{Western blot}

The cell proteins in the experimental group and the control group were extracted. After the concentration of proteins was determined, the proteins were separated with polyacrylamide gel electrophoresis. The primary (Anti-LC3, 1:1,000, cat. no. 14796S, Cell Signaling Technology, Inc.) and secondary antibodies (1:5000; cat. no. AA24142, Cell Signaling Technology, Inc.) were added to the membranes, and imaging was performed after the immune response was completed. The signals were detected using enhanced chemiluminescence reagent (GE Healthcare) and Image J software (version 146; National Institutes of Health, Bethesda, MD, USA) was used to analyze the fold-changes of protein levels, and the relative expression level of the protein was determined by the ratio of the target fragment to the internal reference ( $\beta$-actin).

\section{Immunofluorescence}

The cells were fixed with cold acetone for $10 \mathrm{~min}$ and then washed with PBS. The cells were then sectioned and a Vectastain ABC Kit (Vector Laboratories, USA) was used for immunohistochemical analysis according to the instructions. And the sections were incubated with normal blocking serum and the primary antibodies LC3 (ab48394; abcam, USA) overnight. The next day, after cleaning, the sections were incubated for $1 \mathrm{~h}$ at room temperature with a biotinylated secondary antibody (ab150077; abcam, USA). After that, the labeled cell sections were fluorescently photographed under the Olympus BX51 microscope.

\section{Statistical analysis}

The data were expressed as the mean \pm standard deviation (SD). The statistical analysis was carried out using one-way analysis of variance (ANOVA) followed by Tukey's post hoc-tests using the SPSS 22.0 (IBM Corp.). $p<0.05$ was considered to indicate statistically significant differences. All experiments were performed in triplicate.

\section{Results}

\section{PM2.5 inhibits the proliferation of human epidermal melanocytes}

Different concentrations $(1,10,100$ and $250 \mu \mathrm{g} / \mathrm{ml})$ of W-PM2.5 and NW-PM2.5 poisoning venom were used to treat human epidermal melanocytes. CCK- 8 was used to measure cell proliferation rate. The results showed that with the increasing concentrations of PM2.5, the proliferation rate of melanocytes decreased significantly, and the proliferation rate of melanocytes in NW-PM2.5 group was greater than that in the W-PM2.5 group (Fig. 1A). Subsequently, we further tested whether the decreased cell survival rate in the NW-PM2.5 group was caused by DMSO. By calculation, we found that the concentration of DMSO in $1 \mu \mathrm{g} / \mathrm{ml}$ and $10 \mu \mathrm{g} / \mathrm{ml}$ of NW-PM2.5 was less than $1 \%$, so we will not verify this in the experiment. We mainly verified whether the concentration of DMSO in $100 \mu \mathrm{g} / \mathrm{ml}$ and $250 \mu \mathrm{g} / \mathrm{ml}$ of NW-PM2.5 had an effect on cell survival. We divided the cells into Control, DMSO (DMEM+DMSO of the same amount as $250 \mu \mathrm{g} / \mathrm{ml}$ or $100 \mu \mathrm{g} / \mathrm{ml}$ of NW-PM2.5) and $250 \mu \mathrm{g} / \mathrm{ml}$ or $100 \mu \mathrm{g} / \mathrm{ml}$, groups and then CCK-8 technology detected the cell survival rate. We found that compared with the Control
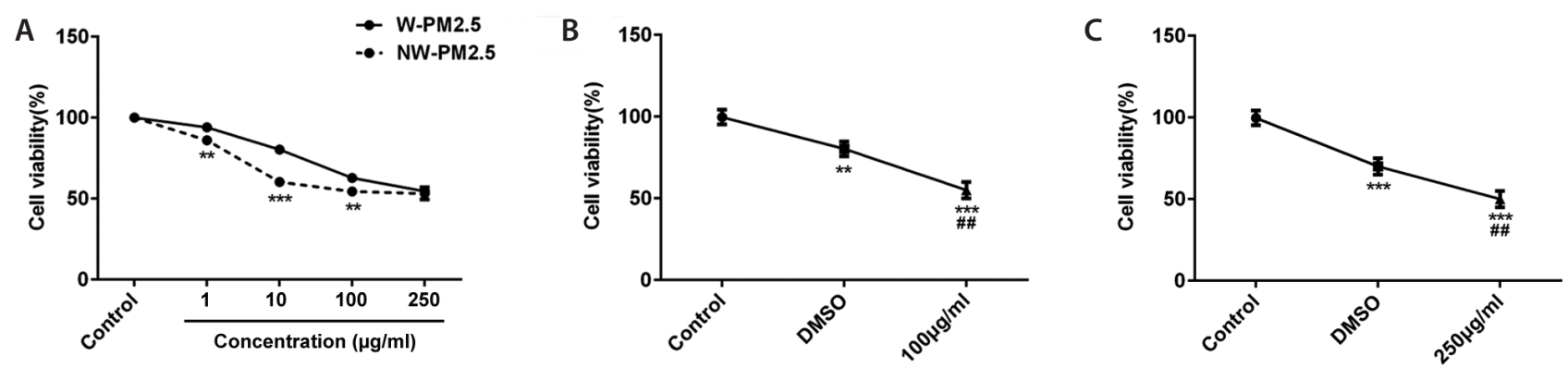

Figure 1. PM2.5 inhibits the proliferation of human epidermal melanocytes. A. CCK- 8 was used to detect the cell viability after being induced by different concentrations $(0,1,10,100$ and $250 \mu \mathrm{g} / \mathrm{ml})$ of PM2.5. B. CCK- 8 was used to detect the cell viability after being induced by DMSO and NW-PM2.5. DMSO, DMEM+DMSO of the same amount as $100 \mu \mathrm{g} / \mathrm{ml}$ of NW-PM2.5; $100 \mu \mathrm{g} / \mathrm{ml}, 100 \mu \mathrm{g} / \mathrm{ml}$ of NW-PM2.5. C. CCK-8 was used to detect the cell viability after being induced by DMSO and NW-PM2.5. DMSO, DMEM+DMSO of the same amount as $250 \mu \mathrm{g} / \mathrm{ml}$ of NW-PM2.5; $250 \mu \mathrm{g} / \mathrm{ml}, 250 \mu \mathrm{g} / \mathrm{ml}$ of NW-PM2.5. ${ }^{* *} p<0.01,{ }^{* * *} p<0.001 v$ s. Control group; ${ }^{\# \#} p<$ 0.01 vs. DMSO group. 
A
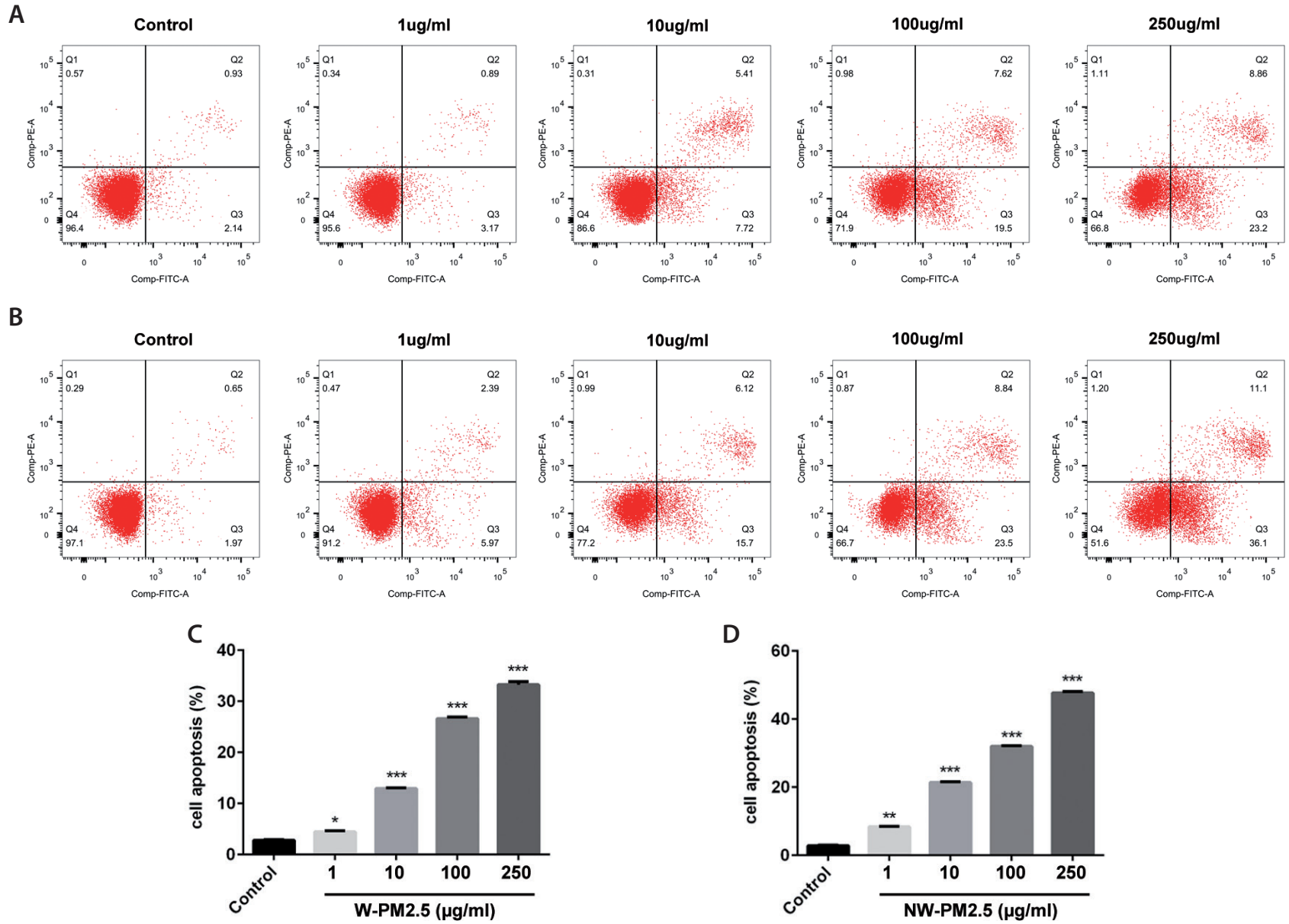

Figure 2. PM2.5 promotes cell apoptosis of human epidermal melanocytes. The apoptosis rate of melanocytes in W-PM2.5 group (A) and NW-PM2.5 group (B) was detected by flow cytometry. C, D. The statistical analysis diagram of A and B, respectively. ${ }^{\star} p<0.05$, ${ }^{* *} p<0.01,{ }^{* * *} p<0.001 v s$. Control group.

group, the survival rate of DMSO group decreased slightly. Compared with the DMSO group, the cell survival rate of the $250 \mu \mathrm{g} / \mathrm{ml}$ (Fig. 1B) and $100 \mu \mathrm{g} / \mathrm{ml}$ (Fig. 1C) groups significantly decreased, indicating that NW-PM2.5 can promote cell death. The results showed that PM2.5 could inhibit cell proliferation.

\section{PM2.5 promotes the apoptosis of human epidermal melanocytes}

Then we further examined the effect of PM2.5 on apoptosis by flow cytometry. As shown in Figure 2A and C, with the increasing concentrations of W-PM2.5, the apoptosis rate of melanocytes increased significantly. In NW-PM2.5 group, PM2.5 concentration of $1,10,100,250 \mu \mathrm{g} / \mathrm{ml}$ can obviously induce cell apoptosis in a concentration-dependent manner compared with the Control group (Fig. 2B and D). The results showed that PM2.5 could inhibit cell proliferation and promote cell apoptosis.
PM2.5 can induce oxidative stress in human epidermal melanocytes

In order to explore the mechanism where PM2.5 induced cell apoptosis, we examined the levels of indicators related to oxidative stress. We found that after PM2.5 induction, the expression of ROS in melanocytes increased significantly in a dose-dependent manner both in W-PM2.5 group (Fig. 3A) and NW-PM2.5 group (Fig. 3B). It suggests that PM2.5 may induce oxidative stress in human epidermal melanocytes, and thus promote apoptosis.

\section{PM2.5 induces autophagy in human epidermal melanocytes}

The increase of ROS expression is generally accompanied by an increase in autophagy, so we measured the expression level of autophagy in cells induced by PM2.5. As shown in Fig. $4 \mathrm{~A}$ and $\mathrm{B}$, the melanin nuclei of melanocytes are of dif- 
A

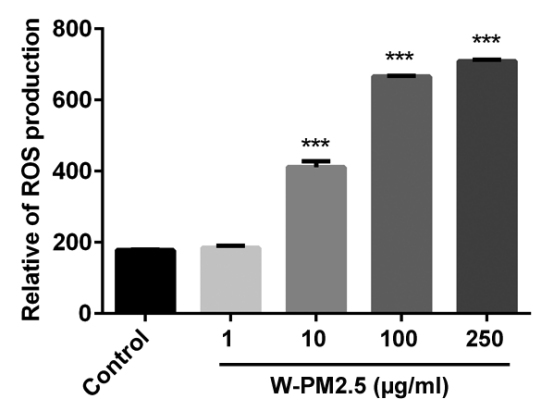

B

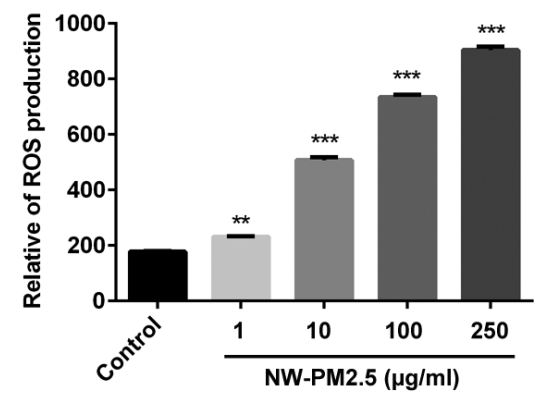

Figure 3. PM2.5 can induce oxidative stress in human epidermal melanocytes. The expression of ROS in W-PM2.5 group (A) and NW-PM2.5 group (B) was detected by ELISA-test. ${ }^{* *} p<0.01,{ }^{* * *} p<$ 0.001 vs. Control group. ferent sizes under fluorescence microscopy, and the bright green particles are autophagosomes. It can be seen that with the increasing concentrations of W-PM2.5 (Fig. 4A and C) and NW-PM2.5 (Fig. 4B and D), the bright green autophagosomes in melanocytes and the fluorescence intensity increased significantly. At the same concentration, the number and fluorescence intensity of autophagosomes in the NW-PM2.5 group were significantly higher than those

A
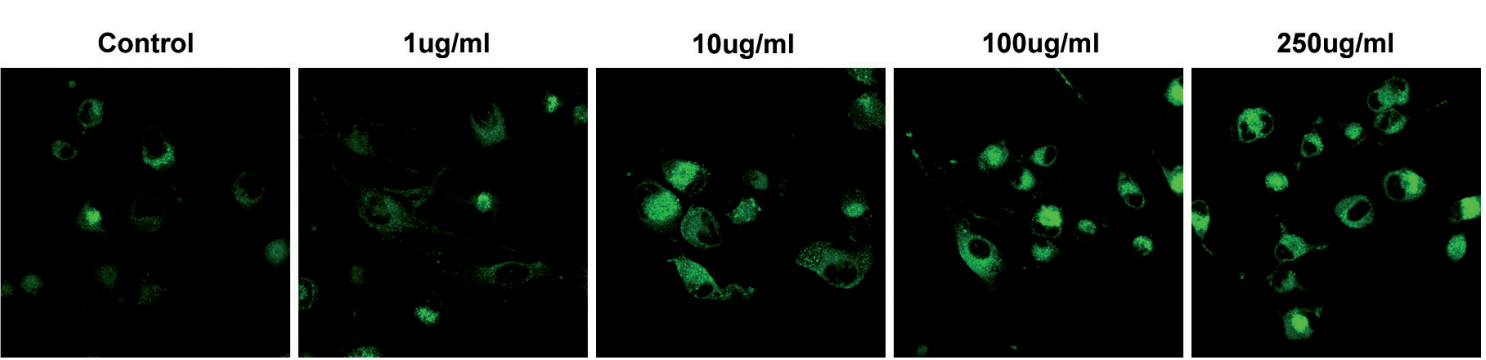

B
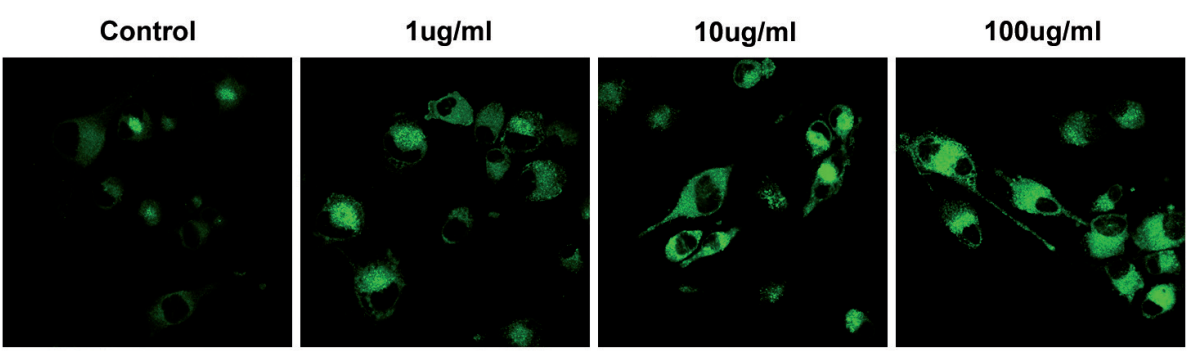

$250 \mathrm{ug} / \mathrm{ml}$

C

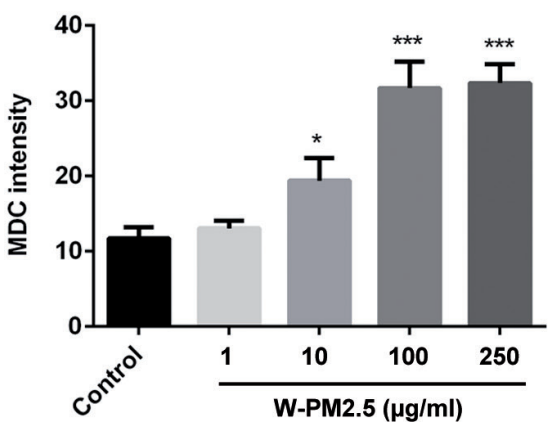

D

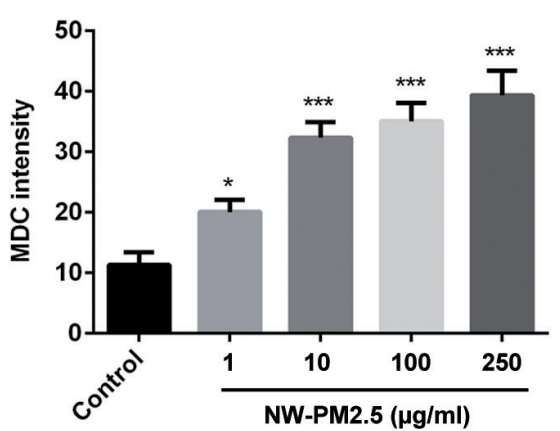

Figure 4. PM2.5 induces autophagy in human epidermal melanocytes. Expressions of autophagosomes in W-PM2.5 group (A) and NW-PM2.5 group (B) were detected by MDC staining. The scale was $20 \mu \mathrm{m}$. C, D. Cell quantification in MDC staining experiments. The bright green particles are autophagosomes (for color figures see online version of the manuscript). ${ }^{*} p<0.05,{ }^{* *} p<0.001 v s$. Control group. 
A
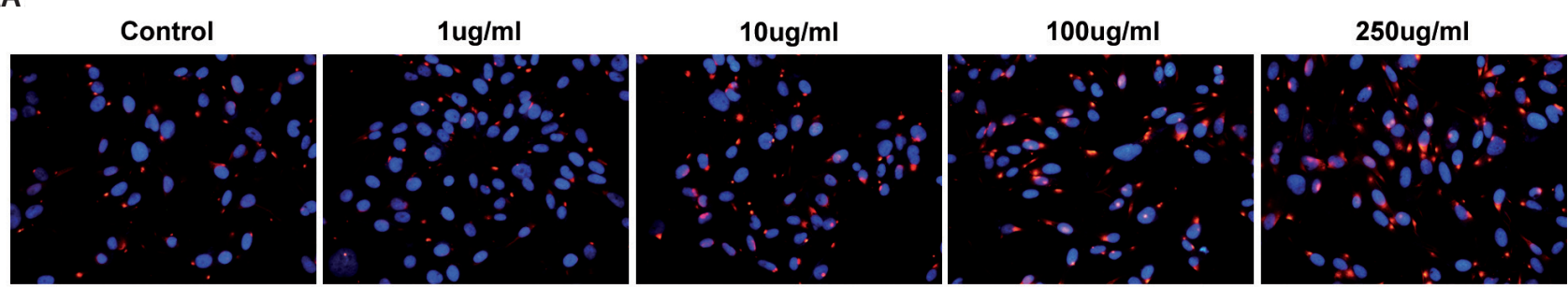

B
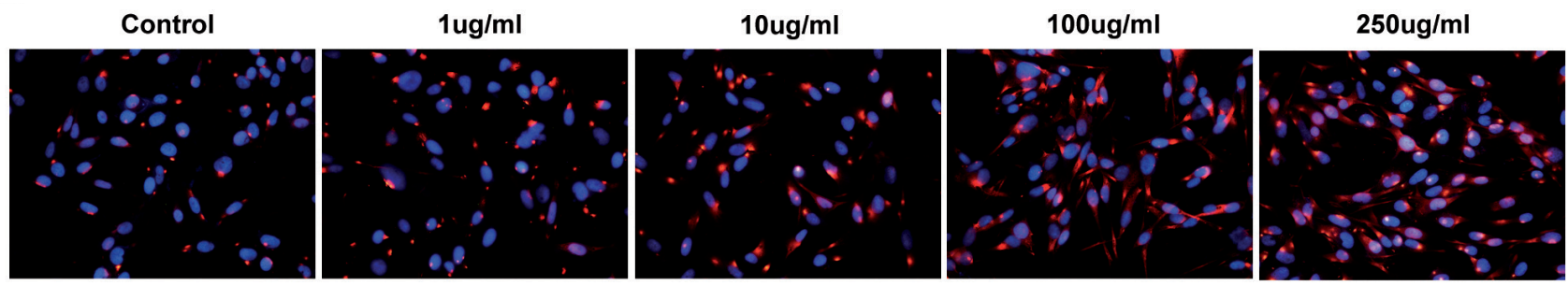

C

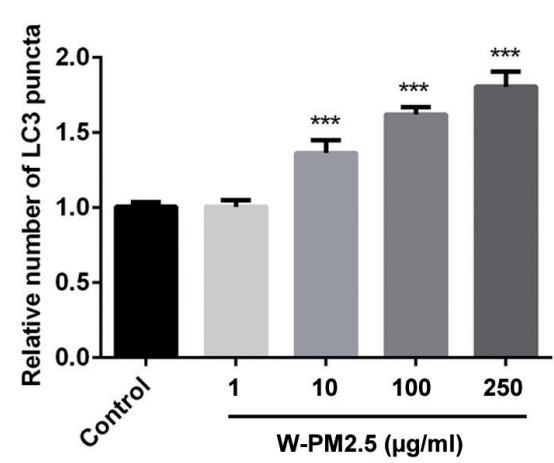

D

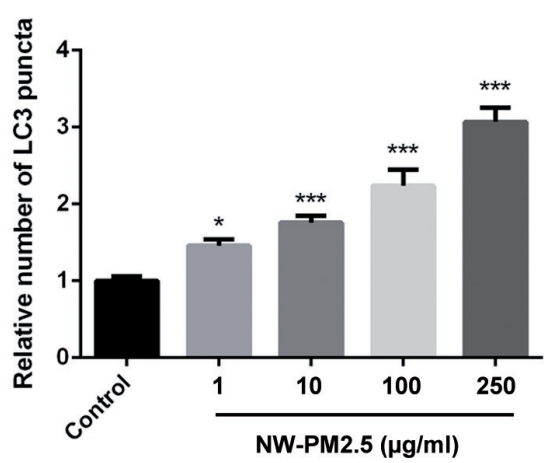

Figure 5. PM2.5 induces autophagy in human epidermal melanocytes. The expression of autophagy marker protein LC3 in W-PM2.5 group (A) and NW-PM2.5 group (B) was detected by immunohistochemistry. C, D. Relative quantification of cells in immunofluorescence staining assay. Blue fluorescence indicated nuclear staining and red fluorescence indicated LC3 staining (for color figures see online version of the manuscript). ${ }^{\star} p<0.05,{ }^{* *} p<0.001 v s$. Control group.

in the W-PM2.5 group. Immunohistochemistry technique was used to detect the expression of autophagy marker protein LC3. It can be seen that LC3 expression was very weak in the control group. However, in the experimental group treated with different concentrations of W-PM2.5 (Fig. 5A and C) and NW-PM2.5 (Fig. 5B and D) at 1, 10, 100 , and $250 \mu \mathrm{g} / \mathrm{ml}$, LC3 showed positive or even strong positive expression after fluorescence staining, and its red fluorescence intensity increased with the increasing concentration of the toxic solution, indicating that the level of autophagy increased significantly. Subsequently, the expression of LC3 was further detected by Western blot. We found that compared with the control group, the expressions of LC3 in the remaining groups increased with the increasing concentrations of PM2.5 both in W-PM2.5 group and NWPM2.5 group (Fig. 6). Moreover, it can be seen that with the same concentration, NW-PM2.5 has a stronger promoting effect on LC3 expression than W-PM2.5 group. The results showed that PM2.5 could induce excessive autophagy of human epidermal melanocytes, thus triggering apoptosis.

\section{Discussion}

Nowadays, there are more and more studies on the harm of PM2.5 to cardiovascular system and respiratory system, but there are few studies discussing about the harm of PM2.5 to skin (Pun et al. 2017; Li et al. 2018c). Therefore, in this paper, human epidermal melanocytes were selected and treated with different concentrations of PM2.5 in vitro to study the specific effect of PM2.5 on human epidermal melanocytes. Our experimental results showed that PM2.5 can inhibit the proliferation of human epidermal melanocytes and promote apoptosis. That is to say, PM2.5 can cause cellular damage to melanocytes, but the specific mechanism is not clear. 

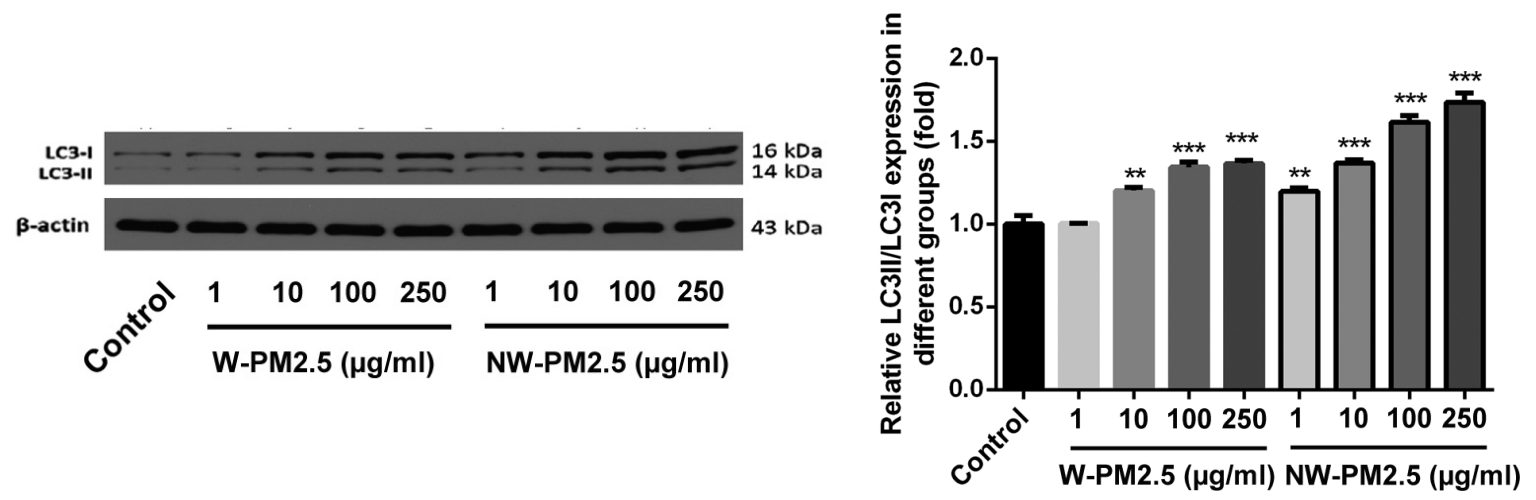

Figure 6. PM2.5 induces the expression of autophagy-related protein in human epidermal melanocytes. The expression of autophagy marker protein LC3 was detected by Western blot. ${ }^{* *} p<0.01,{ }^{* * *} p<0.001$ vs. Control group.

Studies on the pathogenesis of PM2.5 have shown that oxidative damage is one of the most common and important determinants of its toxicity to cells that will ultimately lead to different pathological damage (Chenxu et al. 2018; Li et al. 2018a, 2018b). The surface of PM2.5 is rich in metal ions such as iron, copper, zinc and manganese, as well as organic compounds such as polycyclic aromatic hydrocarbons (PAHs), which can stimulate the body to generate free radicals, consume antioxidant components and cause oxidative stress (Feng et al. 2016). Long et al. (2019) showed that PM2.5 can induce the production of ROS in vascular endothelial cells, and lead to oxidative stress, including decreased superoxide dismutase activity, release of lactate dehydrogenase, and increased membrane permeability. Besides, Wang et al. (2019a) reported that PM2.5 inhibits SOD1 expression by upregulating microRNA-206 and promotes ROS accumulation and disease progression in asthmatic mice. Our results also showed that the expressions of oxidative stress-related factors in melanocytes were significantly increased after PM2.5 treatment. It indicated that PM2.5 can cause oxidative stress reaction in melanocytes and induce stress damage in cells.

Oxidative stress can induce autophagy by producing a large amount of ROS (Li et al. 2015). Autophagy refers to the process in which the intracellular substrates degraded in the cell (such as organelles, proteins, etc.) are wrapped in a double-layer membrane structure to form autophagosomes and transport to lysosomes for membrane fusion (Gao 2019). A series of hydrolases in lysosomes digest the cell's own proteins or organelles to maintain cell metabolism and the regeneration of some organelles (Yu et al. 2018). Autophagy is mediated by a series of autophagy-related genes, among which the expression of LC3II/I is a representative indicator of the intensity of autophagy (Schaaf et al. 2016). Some scholars believe that autophagy is the basic stress response of cells to ensure cell survival under harsh conditions. Modest autophagy activities can timely remove damaged organelles, proteins and other harmful components produced by damaged cells, provide emergency substrates and energy, and gain time and conditions to repair damaged DNA (Parzych and Klionsky 2014). However, excessive autophagy will induce programmed cell death (Bialik et al. 2018). Therefore, autophagy can not only promote the health of the body, but also be closely related to the pathogenesis of tumors, infectious diseases and immune diseases (Saha et al. 2018).

Studies have shown that the autophagy process of deficient melanocytes is similar to that of melanocytes from skin lesions in patients with vitiligo, suggesting that abnormal melanocyte autophagy may be associated with the onset of vitiligo (Yang et al. 2018). In addition, autophagy can regulate the formation of melanin in melanocytes and the generation of melanosome to adjust the skin color of the body (Kim et al. 2019). Hirone and Eyru (1978) observed skin lesions of patients with simple freckles by electron microscopy. They also found that large granules of pigment generated by the autophagy of melanocytes occurred in melanocytes and corneum cells. After $0.05 \%$ of $\mathrm{H}_{2} \mathrm{O}_{2}$ induction, the autophagy of human melanocyte proceeded, with the result of decreased dendritic retraction rate of melanocyte, and increased expressions of autophagy-related proteins - Beclin1 and LC3II/I. Gong et al. therefore, speculated that the autophagy pathway could protect melanocytes from damage (Gong et al. 2015). These studies indicate that autophagy plays an important role in the pathogenesis of pigmentary dermatosis. We detected the expression of autophagy in PM2.5-induced melanocytes. We found that after PM2.5 induction, the expression of autophagosome and autophagy marker protein LC3-II /I in melanocytes increased, and the autophagy response was significantly enhanced. Moreover, at the same concentration, the effects of NW-PM2.5 and W-PM2.5 on cell proliferation, apoptosis and autophagy are different. Which one has the greatest influence and what is the mechanism? Our laboratory will further study it in the following experiments. 


\section{Conclusion}

In conclusion, our experimental results showed that PM2.5 can inhibit the proliferation of human epidermal melanocytes and promote apoptosis, which may be related to the fact that PM2.5 can induce oxidative stress injury and excessive autophagy of melanocytes.

Acknowledgements. Not applicable.

Funding. Health Commission of Hubei Province scientific research project, WJ2019M212.

Availability of data and materials. The analyzed data sets generated during the present study are available from the corresponding author on reasonable request.

Conflict of interests. The authors declare that they have no competing interests.

\section{References}

Bialik S, Dasari SK, Kimchi A (2018): Autophagy-dependent cell death - where, how and why a cell eats itself to death. J. Cell. Sci. 131, jcs215152 https://doi.org/10.1242/jcs.215152

Chenxu G, Minxuan X, Yuting Q, Tingting G, Jinxiao L, Mingxing W, Sujun W, Yongjie M, Deshuai L, Qiang L, et al. (2018): iRhom 2 loss alleviates renal injury in long-term PM2.5-exposed mice by suppression of inflammation and oxidative stress. Redox. Biol. 19, 147-157 https://doi.org/10.1016/j.redox.2018.08.009

Feng S, Gao D, Liao F, Zhou F, Wang X (2016): The health effects of ambient PM2.5 and potential mechanisms. Ecotoxicol. Environ. Saf. 128, 67-74 https://doi.org/10.1016/j.ecoenv.2016.01.030

Galvao ES, D'azeredo Orlando MT, Santos JM, Lima AT (2020): Uncommon chemical species in PM2.5 and PM10 and its potential use as industrial and vehicular markers for source apportionment studies. Chemosphere 240, 124953 https://doi.org/10.1016/j.chemosphere.2019.124953

Gao Q (2019): Oxidative stress and autophagy. Adv. Exp. Med. Biol. 1206, 179-198

https://doi.org/10.1007/978-981-15-0602-4_9

Glassman SJ (2011): Vitiligo, reactive oxygen species and T-cells. Clin. Sci. (Lond) 120, 99-120 https://doi.org/10.1042/CS20090603

Gong Q, Li X, Sun J, Ding G, Zhou M, Zhao W, Lu Y (2015): The effects of calcipotriol on the dendritic morphology of human melanocytes under oxidative stress and a possible mechanism: is it a mitochondrial protector? J. Dermatol. Sci. 77, 117-124 https://doi.org/10.1016/j.jdermsci.2014.12.006

Hirone T, Eryu Y (1978): Ultrastructure of giant pigment granules in lentigo simplex. Acta Derm. Venereol. 58, 223-229

Kim JY, Kim J, Ahn Y, Lee EJ, Hwang S, Almurayshid A, Park K, Chung HJ, Kim HJ, Lee SH, et al. (2019): Autophagy induc- tion can regulate skin pigmentation by causing melanosome degradation in keratinocytes and melanocytes. Pigment Cell Melanoma Res. 33, 403-415

https://doi.org/10.1111/pcmr.12838

Kupsa R, Narro-Bartenstein E, Hofmann-Wellenhof R (2019): Suspicious hyperpigmentation with parallel ridge pattern on acral skin: Three case reports. Hautarzt 71, 154-157 (in German) https://doi.org/10.1007/s00105-019-04511-3

Li J, Zhou Q, Liang Y, Pan W, Bei Y, Zhang Y, Wang J, Jiao Z (2018a): miR-486 inhibits PM2.5-induced apoptosis and oxidative stress in human lung alveolar epithelial A549 cells. Ann. Transl. Med. 6, 209

https://doi.org/10.21037/atm.2018.06.09

Li J, Zhou Q, Yang T, Li Y, Zhang Y, Wang J, Jiao Z (2018b): SGK1 inhibits PM2.5-induced apoptosis and oxidative stress in human lung alveolar epithelial A549cells. Biochem. Biophys. Res. Commun. 496, 1291-1295 https://doi.org/10.1016/j.bbrc.2018.02.002

Li L, Tan J, Miao Y, Lei P, Zhang Q (2015): ROS and autophagy: interactions and molecular regulatory mechanisms. Cell. Mol. Neurobiol. 35, 615-621 https://doi.org/10.1007/s10571-015-0166-x

Li R, Zhou R, Zhang J (2018c): Function of PM2.5 in the pathogenesis of lung cancer and chronic airway inflammatory diseases. Oncol. Lett. 15, 7506-7514 https://doi.org/10.3892/ol.2018.8355

Liu Q, Lu Z, Xiong Y, Huang F, Zhou J, Schauer JJ (2020): Oxidative potential of ambient PM2.5 in Wuhan and its comparisons with eight areas of China. Sci. Total. Environ. 701, 134844 https://doi.org/10.1016/j.scitotenv.2019.134844

Long YM, Yang XZ, Yang QQ, Clermont AC, Yin YG, Liu GL, Hu LG, Liu Q, Zhou QF, Liu QS, et al. (2019): PM2.5 induces vascular permeability increase through activating MAPK/ ERK signaling pathway and ROS generation. J. Hazard Mater. 2019, 121659 https://doi.org/10.1016/j.jhazmat.2019.121659

Mannucci PM, Harari S, Franchini M (2019): Novel evidence for a greater burden of ambient air pollution on cardiovascular disease. Haematologica 104, 2349-2357 https://doi.org/10.3324/haematol.2019.225086

Ning J, Li P, Zhang B, Han B, Su X, Wang Q, Wang X, Li B, Kang H, Zhou L, et al. (2019): miRNAs deregulation in serum of mice is associated with lung cancer related pathway deregulation induced by PM2.5. Environ. Pollut. 254, 112875 https://doi.org/10.1016/j.envpol.2019.07.043

Parzych KR, Klionsky DJ (2014): An overview of autophagy: morphology, mechanism, and regulation. Antioxid. Redox. Signal. 20, 460-473 https://doi.org/10.1089/ars.2013.5371

Pun VC, Kazemiparkouhi F, Manjourides J, Suh HH (2017): Long-term PM2.5 exposure and respiratory, cancer, and cardiovascular mortality in older US adults. Am. J. Epidemiol. 186, 961-969 https://doi.org/10.1093/aje/kwx166

Saha S, Panigrahi DP, Patil S, Bhutia SK (2018): Autophagy in health and disease: A comprehensive review. Biomed. Pharmacother. 104, $485-495$ 
https://doi.org/10.1016/j.biopha.2018.05.007

Schaaf MB, Keulers TG, Vooijs MA, Rouschop KM (2016): LC3/ GABARAP family proteins: autophagy- (un)related functions. FASEB J. 30, 3961-3978 https://doi.org/10.1096/fj.201600698R

Su SY, Liaw YP, Jhuang JR, Hsu SY, Chiang CJ, Yang YW, Lee WC (2019): Associations between ambient air pollution and cancer incidence in Taiwan: an ecological study of geographical variations. BMC Public Health 19, 1496 https://doi.org/10.1186/s12889-019-7849-z

Wan Q, Liu Z, Yang M, Wu J (2019): Acceleratory effects of ambient fine particulate matter on the development and progression of atherosclerosis in apolipoprotein E knockout mice by downregulating CD4 $(+) \mathrm{CD} 25(+)$ Foxp3 $(+)$ regulatory T cells. Toxicol. Lett. 316, 27-34 https://doi.org/10.1016/j.toxlet.2019.09.005

Wang L, Xu J, Liu H, Li J, Hao H (2019a): PM2.5 inhibits SOD1 expression by up-regulating microRNA-206 and promotes ROS accumulation and disease progression in asthmatic mice. Int. Immunopharmacol. 76, 105871 https://doi.org/10.1016/j.intimp.2019.105871

Wang N, Mengersen K, Tong S, Kimlin M, Zhou M, Wang L, Yin P, Xu Z, Cheng J, Zhang Y, Hu W (2019b): Short-term association between ambient air pollution and lung cancer mortality. Environ. Res. 179, 108748

https://doi.org/10.1016/j.envres.2019.108748
Yang F, Yang L, Wataya-Kaneda M, Haswgawa J, Yoshimori T, Tanemura A, Tsurida D, Katayama I (2018): Dysregulation of autophagy in melanocytes contributes to hypopigmented macules in tuberous sclerosis complex. J. Dermatol. Sci. 89, 155-164 https://doi.org/10.1016/j.jdermsci.2017.11.002

Yang SI, Kim HB, Kim HC, Lee SY, Kang MJ, Cho HJ, Yoon J, Jung S, Lee E, Yang HJ, et al. (2020): Particulate matter at third trimester and respiratory infection in infants, modified by GSTM1. Pediatr. Pulmonol. 55, 245-253 https://doi.org/10.1002/ppul.24575

Yang Y, Yang T, Liu S, Cao Z, Zhao Y, Su X, Liao Z, Teng X, Hua J (2019): Concentrated ambient PM2.5 exposure affects mice sperm quality and testosterone biosynthesis. PeerJ 7, e8109 https://doi.org/10.7717/peerj.8109

Yu L, Chen Y, Tooze SA (2018): Autophagy pathway: Cellular and molecular mechanisms. Autophagy 14, 207-215 https://doi.org/10.1080/15548627.2017.1378838

Zhang Y, Chu M, Zhang J, Duan J, Hu D, Zhang W, Yang X, Jia X, Deng F, Sun Z (2019): Urine metabolites associated with cardiovascular effects from exposure of size-fractioned particulate matter in a subway environment: A randomized crossover study. Environ. Int. 130, 104920 https://doi.org/10.1016/j.envint.2019.104920

Received: February 12, 2020

Final version accepted: May 19, 2020 